\title{
SOBREPESO E OBESIDADE EM ALUNOS DE 6-10 ANOS DE ESCOLA ESTADUAL DE UMUARAMA/PR
}

\author{
Adryangela Renata Martins de Carvalho ${ }^{1}$ \\ Mônica de Oliveira Belém² \\ Juliano Yasuo Oda ${ }^{3}$
}

CARVALHO, A. R. M de; BELÉM, M. de O.; ODA. J. Y. Sobrepeso e obesidade em alunos de 6-10 anos de escola Estadual de Umuarama/ PR. Arq. Cienc. Saúde UNIPAR, Umuarama, v. 21, n. 1, p, 3-12, jan./abr. 2017.

\begin{abstract}
RESUMO: A obesidade e o sobrepeso vem crescendo de forma acentuada, principalmente na infância e na adolescência, tornando-se um grave problema de saúde pública. A obesidade infantil está crescendo acentuadamente em vários países incluindo o Brasil, sendo importante fator de risco para o desenvolvimento das doenças cardiovasculares na vida adulta. O acúmulo de tecido adiposo, sobretudo abdominal, é fundamental para o desencadeamento da síndrome metabólica (SM). Objetivou-se identificar o perfil antropométrico e os fatores predisponentes da SM. Foi realizado um questionário, estudos antropométrico e nutricional de crianças de 6-10 anos de escola estadual de Umuarama/PR. Foi estudado um total de 226 crianças com média das idades de 8,68 anos, sendo a maioria meninas na faixa de 9 anos. Avaliou-se sobrepeso e obesidade entre as crianças de ambos os sexos, bem como a deficiência de atividade física e hábitos alimentares pouco saudáveis, favorecendo o aumento do IMC e score Z das crianças. Sendo assim, torna-se importante maior incentivo na qualidade e quantidade na atividade física e também orientações relacionadas à alimentação saudável, prevenido que essas crianças se tornem adultos obesos, diminuindo os riscos de desenvolverem a SM, além de evitar os problemas psicológicos gerados pela discriminação relacionada com o excesso de peso, que podem gerar ansiedade, depressão e até mesmo o isolamento social. Fica claro que o envolvimento familiar, juntamente com a escola torna-se essencial para o êxito da prevenção e tratamento da obesidade.
\end{abstract}

PALAVRAS-CHAVE: Índice de Massa Corpórea. Obesidade Infantil. Síndrome Metabólica.

\section{OVERWEIGHT AND OBESITY IN STUDENTS AGED 6-10 YEARS IN A STATE SCHOOL IN UMUARAMA/PR}

\begin{abstract}
Obesity and overweight rates have been growing fast, mainly in childhood and adolescence, and are becoming a serious public health issue. Childhood obesity is sharply rising in several countries, including Brazil. It is also an important risk factor for the development of cardiovascular diseases in adulthood. The accumulation of fat, especially abdominal, is essential to trigger metabolic syndromes (MS). This study aimed at identifying the anthropometric profile and predisposing factors of MS. A questionnaire and an anthropometric and nutritional assessment were applied to children aged 6 to 10 years in a state school in Umuarama/PR. A total of 226 children with mean age of 8.68 years were studied, most of them being girls in the age range of 9 years old. Overweight and obesity were assessed among children of both genders, as well as the lack of physical activity and unhealthy eating habits favoring an increased BMI and Z score in the children. Therefore, it is important to have more incentive towards the quality and quantity of physical activities, and also guidance related to healthy eating, preventing these children to become obese adults, decreasing the risk of developing MS and avoiding the psychological problems generated by social discrimination regarding obesity, which can lead to anxiety, depression and even social isolation. A close relation between family and schools towards prevention and treating obesity is necessary.
\end{abstract}

KEYWORDS: Body Mass Index. Child obesity. Metabolic syndrome.

\section{Introdução}

A obesidade e o sobrepeso vêm crescendo de forma acentuada principalmente na infância e na adolescência. Esse aumento está se tornando um grave problema de saúde pública, e pode-se dizer uma epidemia mundial. Esse fato é preocupante, pois as crianças e adolescentes com obesidade ou sobrepeso no presente poderão ser os adultos obesos do futuro, podendo resultar na forma mais severa dessa doença e também está associado ao aparecimento de outras enfermidades crônicas, tais como: hipertensão arterial sistêmica, anormalidade lipídicas, resistência a insulina, doença coronariana e diabetes mellitos tipo 2 e contribui para a baixa autoestima e discriminação social, oportunizando, assim, complicações emocionais (LERARIO et al., 2002; OLIVEIRA et al., 2003; OLIVEIRA; FISBERG, 2003; CIOLAC; GUIMARÃES, 2004; RIBEIRO et al., 2006; LEMOS et al., 2007; LOTTENBERG; GLEZER; TURATT, 2007; MONDINI et al., 2007).

Os renomados doutrinadores Halpern; Rodrigues;
Costa (2004), definem obesidade como um estado em que há maior quantidade de tecido adiposo em relação à massa magra do que esperado para o sexo, a idade e a altura. Os fatores que contribuem para essa causa são: os internos que são os genéticos no qual os indivíduos tornam susceptíveis ao ganho de peso se entrarem em contato com fatores do meio que sejam favoráveis a isso; e os metabólicos que se referem ao gasto energético, ou seja, à energia gasta pelo organismo para realizar todo tipo de atividade. Outros fatores que contribuem são os externos como os Alimentares que depende do tipo de alimentos, a forma de preparo e as preferências alimentares; os psicológicos que são os problemas emocionais e os psicológicos que podem levar a criança a comer mais como mecanismo de compensação ou de defesa; e as atividades físicas onde o estilo de vida da criança reflete um fator muito importante no desenvolvimento da obesidade (BARBOSA, 2008; HUGHES et al., 2015; TATE et al., 2015).

De acordo com estudos realizados, a obesidade pode ser dividida em obesidade de origem exógena - a mais

DOI: https://doi.org/10.25110/arqsaude.v21i1.2017.6070

${ }^{1}$ Enfermeira graduada pela Unipar - Universidade Paranaense.

${ }^{2}$ Biomédica, Mestre em Patologia Experimental e Doutoranda do Programa de Pós Graduação em Ciências Médicas pela Universidade Federal do Ceará, Laboratório Escola Luis Capelo (UFC); monica.obelem@gmail.com

${ }^{3}$ Fisioterapeuta,Professor da UFMS - Universidade Federal de Mato Grosso do Sul, Mestre em Anatomia e Doutorando do Programa de Pós Graduação em Patologia Experimental pela Universidade Estadual de Londrina(UEL); Laboratório de Neurogastroenterologia (UEL) jyoda1@gmail.com 
frequente - e endógena. Para a endógena, deve-se identificar a doença básica e tratá-la, pois a obesidade endógena ou secundária é causada por doenças de origens hormonais e/ ou genéticas tais como: alteração do metabolismo tireodiano, gonadal, hipotálamo-hipofisário e tumores como o crâniofaringeoma, acometendo menos de $10 \%$ dos casos, já a obesidade exógena ou nutricional sua etiologia não é orgânica, origina-se do desequilíbrio entre ingestão e gasto calórico, devendo ser manejada com orientação alimentar, especialmente mudanças de hábitos e otimização da atividade física, aproximadamente $90 \%$ dos casos corresponde a ela (FISBERG, 1995; DIETZ, 1999; MÜLLER, 2003; BOUGLÉ; BROUARD, 2013; WELSBY et al., 2015).

No que diz respeito à obesidade infantil percebe-se que ela cresce acentuadamente em vários países incluindo o Brasil (FRIEDRICH et al, 2015), mas o país com maior quantidade de casos ainda é o Estados Unidos. De acordo com de Souza Leão et al. (2003), Brasil, Dinamarca, Itália e Baharein são os países que apresentam a mais rápida elevação na prevalência de sobrepeso e obesidade (avaliados pelo IMC) em crianças e adolescentes, mesmo em populações mais carentes (PÉREZ, 2013). A prevalência da obesidade na infância e adolescência no Brasil apresenta um crescimento rápido nas últimas décadas segundo o Instituto Brasileiro de Geografia e Estatística (IBGE) variam entre 10,8\% a 33,8\% em diferentes regiões.

Oliveira et al. (2004) relatam em seu estudo que a obesidade na infância e adolescência é um importante fator de risco para o desenvolvimento das doenças cardiovasculares na vida futura, (SABIA; SANTOS; RIBEIRO, 2004), observaram em seu estudo que nas idades mais precoces a morbidade não é frequente, mas no adulto a situação é de risco e leva ao aumento da mortalidade, por associação com a doença arteriosclerótica, hipertensão e alterações metabólicas.

O acúmulo de tecido adiposo, principalmente na região abdominal, é fundamental para o desencadeamento da síndrome metabólica (SM) (LEE et al., 2014). Existe uma associação entre o tecido adiposo e as principais células inflamatórias, levando a um aumento da produção de mediadores inflamatórios e a maior liberação de ácidos graxos livres, cujos efeitos se fazem sentir tanto nas células beta das ilhotas de Langerhans e seus receptores como na parede vascular (CHEN et al., 2014). Estudos em crianças e adolescentes comprovam como esses fenômenos começam precocemente (HEPTULLA et al., 2001). A presença de alterações metabólicas (resistência à insulina, dislipidemia, hipertensão, alterações trombogênicas, hiperuricemia) na infância e adolescência pode contribuir para o desenvolvimento deste processo, já que estudos longitudinais clássicos mostram uma forte associação entre o excesso de peso nas primeiras décadas de vida e a alta taxa de morbimortalidade na vida adulta por doenças cardiovasculares (OLIVEIRA et al., 2004; LUCA; IORDACHE, 2013).

Não há unanimidade para definir síndrome metabólica, mas a Organização Mundial da Saúde (OMS) e a Associação Americana de Diabetes definiram como portadores de $\mathrm{SM}$, indivíduos com intolerância à glicose, tolerância normal à glicose com resistência à insulina ou diabetes e mais duas das seguintes alterações: uso de anti-hipertensivos e/ou pressão arterial elevada (>140/90 mmHg), obesidade (índice de massa corpórea $>30 \mathrm{~kg} / \mathrm{m}^{2}$ ) abdominal (relação cintura-quadril $>0,90$ no homem e $>0,80$ na mulher), níveis elevados de triglicérides ( $>150 \mathrm{mg} / \mathrm{dl})$, HDL-colesterol baixo $(<35 \mathrm{mg} / \mathrm{dl}$ no homem e $<39 \mathrm{mg} / \mathrm{dl}$ na mulher) e microalbuminúria (excreção urinária de albumina $>20 \mu \mathrm{g} / \mathrm{min}$ ). A "National Cholesterol Evaluation Program for Adult Treatment Panel III" (ATP III), definiu como portador da síndrome, o indivíduo que apresentar três ou mais das alterações: como obesidade central (circunferência abdominal $>88 \mathrm{~cm}$ para as mulheres e $>102 \mathrm{~cm}$ para os homens), pressão arterial alta ( $>130 / 85$ $\mathrm{mmHg})$, glicemia de jejum $>110 \mathrm{mg} / \mathrm{dl}$, triglicérides $>150$ $\mathrm{mg} / \mathrm{dl}$, HDL-colesterol baixo $(<40 \mathrm{mg} / \mathrm{dl}$ nos homens e $<50$ $\mathrm{mg} / \mathrm{dl}$ nas mulheres) (ADULT TREATMENT PANEL III; 2001).

Evidências científicas revelam que a aterosclerose e a hipertensão arterial são processos patológicos iniciados na infância, e nesta faixa etária são formados os hábitos alimentares e de atividade física (ABRANTES; LEMOUNIER; COLOSIMO, 2002). Ferreira; Oliveira; França (2007) observaram também que, quanto maior o nível de resistência à insulina, maior a quantidade dos fatores de risco presentes nas crianças obesas e maior a predisposição para desenvolver um diagnóstico futuro de SM, DMT2 e DCV.

Com o crescente número de casos de sobrepeso e obesidade na infância torna-se importante o diagnóstico precoce, facilitando o controle e prevenção de novos casos e assim diminuir de forma satisfatória os agravos que a obesidade pode trazer na fase adulta. Diante do apresentado, o objetivo desse trabalho foi identificar o perfil antropométrico e os fatores predisponentes da síndrome metabólica falando da correlação com a sua possível identificação.

\section{Materiais e Métodos}

Foram estudadas 226 crianças entre 6 e 10 anos, sendo 133 do sexo feminino e 93 do sexo masculino no período de agosto a setembro de 2009, em uma escola estadual de Umuarama, PR. Para seleção das crianças, foi enviado aos pais ou responsáveis de todos presentes no primeiro dia da visita à escola o Termo de Consentimento, participaram da pesquisa aqueles que os pais autorizaram e assinaram o termo de Esclarecimento Livre e Esclarecido. Este trabalho foi aprovado pelo Comitê de Ética em Pesquisas Envolvendo Seres Humanos da Universidade Paranaense - UNIPAR.

A avaliação antropométrica foi realizada com as crianças usando o mínimo de roupas possível sem calçados, sendo que as medidas antropométricas realizadas foram peso e a estatura. Para a verificação do peso empregou - se uma balança ponteira da marca G-tech com capacidade de até 130 $\mathrm{kg}$ colocada sobre superfície plana, e a estatura foi mensurada com fita métrica inextensível (fixada em parede lisa).

Foi aplicado um questionário contendo 10 questões fechadas na qual abordava assunto relacionado aos hábitos alimentares e de atividade física, assim facilitando a identificação do provável motivo do estado nutricional das crianças.

$\mathrm{Na}$ avaliação do estado nutricional, foi adotado o critério da Organização mundial de saúde (WHO, 1998) que estabelece as condições nutricionais de acordo com o percentil ocupado pelo valor do Índice de Massa Corpórea (IMC) foi realizado para ambos os sexos; as crianças foram classificadas com baixo peso (criança abaixo do percentil $5^{\circ}$ ), eu- 
tróficas (percentil $5^{\circ}$ a $85^{\circ}$ ) e obesidade (acima do percentil $95^{\circ}$ ), foram realizadas análise estatística com a Análise de Variância e os valores de escores z para peso/idade, peso/estatura e idade/estatura (WHO, 1995), em que o peso e altura ideais para cada idade foram coletados por meio da tabela do NCHS, dos resultados obtidos para sexos e faixa etária foi calculado o valor médio para faixa etária estudadas.

\section{Resultados}

Foi estudado um total de 226 crianças participantes e a média das idades foi de 8,68 anos, sendo que o número maior de crianças do gênero feminino na faixa de 9 anos. $\mathrm{Na}$
Tabela 1, será observado a distribuição quanto à idade e o estado nutricional das crianças do sexo masculino por meio do IMC. Encontra-se 18 (19,35\%) de meninos com déficit nutricional (IMC < 10), 17 (18,25\%) estavam apresentando sobrepeso $(10<\mathrm{IMC}<90)$ e $20(21,5 \%)$ estavam apresentaram um quadro de obesidade (IMC $>90$ ). Na Tabela 2 , está representado o estado nutricional das crianças do sexo feminino através IMC. De acordo com os resultados encontrados, 29 $(21,8 \%)$ das meninas estavam apresentaram déficits nutricionais $(\mathrm{IMC}<10) 22(16,54 \%)$ apresentaram estados nutricionais entre sobrepeso $(10<\mathrm{IMC}<90)$ e $31(23,3 \%)$ apresentaram um quadro de obesidade (IMC $>90$ ).

Tabela 1: Distribuição dos escolares do gênero masculino de acordo com os pontos de corte do índice de massa corporal (IMC).

\begin{tabular}{|c|c|c|c|c|c|c|c|c|c|c|}
\hline \multicolumn{11}{|c|}{ IMC } \\
\hline \multirow[t]{2}{*}{ IDADE } & \multicolumn{2}{|c|}{ DISTRIBUIÇÃO } & \multicolumn{2}{|c|}{ BAIXO PESO } & \multicolumn{2}{|c|}{ EUTROFIA } & \multicolumn{2}{|c|}{ SOBREPESO } & \multicolumn{2}{|c|}{ OBESIDADE } \\
\hline & $\mathbf{N}$ & $\%$ & $\mathbf{N}$ & $\%$ & $\mathbf{N}$ & $\%$ & $\mathbf{N}$ & $\%$ & $\mathbf{N}$ & $\%$ \\
\hline 6 ANOS & 8 & 3,53 & 1 & 1,07 & 3 & 3,22 & - & 0 & 4 & 4,3 \\
\hline 7 ANOS & 18 & 7,69 & 2 & 2,15 & 9 & 9,67 & 1 & 1,07 & 6 & 6,45 \\
\hline 8 ANOS & 19 & 8,4 & 3 & 3,22 & 2 & 2,15 & 8 & 8,6 & 6 & 6,45 \\
\hline 9 ANOS & 27 & 11,94 & 7 & 7,52 & 13 & 13,98 & 5 & 5,37 & 2 & 2,15 \\
\hline 10 ANOS & 21 & 9,29 & 5 & 5,37 & 11 & 11,83 & 3 & 3,22 & 2 & 2,15 \\
\hline TOTAL & 93 & 41,15 & 18 & 19,35 & 38 & 40,86 & 17 & 18,27 & 20 & 21,5 \\
\hline
\end{tabular}

Tabela 2: Distribuição dos escolares do gênero feminino de acordo com os pontos de corte do índice de massa corporal (IMC).

\begin{tabular}{|c|c|c|c|c|c|c|c|c|c|c|}
\hline \multicolumn{11}{|c|}{ IMC } \\
\hline \multirow[t]{2}{*}{ IDADE } & \multicolumn{2}{|c|}{ DISTRIBUIÇÃO } & \multicolumn{2}{|c|}{ BAIXO PESO } & \multicolumn{2}{|c|}{ EUTROFIA } & \multicolumn{2}{|c|}{ SOBREPESO } & \multicolumn{2}{|c|}{ OBESIDADE } \\
\hline & $\mathbf{N}$ & $\%$ & $\mathbf{N}$ & $\%$ & $\mathbf{N}$ & $\%$ & $\mathbf{N}$ & $\%$ & $\mathbf{N}$ & $\%$ \\
\hline 6 ANOS & 11 & 4,86 & 1 & 0,75 & 4 & 3 & 2 & 1,5 & 4 & 3 \\
\hline 7 ANOS & 34 & 15 & 4 & 3 & 14 & 10,52 & 8 & 6,01 & 8 & 6,01 \\
\hline 8 ANOS & 31 & 13,71 & 6 & 4,51 & 9 & 6,76 & 6 & 4,51 & 10 & 7,52 \\
\hline 9 ANOS & 35 & 15,48 & 8 & 6,01 & 16 & 12,03 & 5 & 3,76 & 6 & 4,51 \\
\hline 10 ANOS & 22 & 9,73 & 10 & 7,52 & 8 & 6,01 & 1 & 0,75 & 3 & 2,25 \\
\hline TOTAL & 133 & 58,84 & 29 & 21,8 & 51 & 38,34 & 22 & 16,54 & 31 & 23,3 \\
\hline
\end{tabular}

Na Tabela 3, está descrito a distribuição das crianças do gênero masculino por meio do índice peso para a idade em escores z. Na pesquisa foi possível identificar que 4 meninos (4,29\%) apresentaram déficit de peso para a idade (abaixo de
$-2,00$ escores $\mathrm{z}), 16(17,2 \%)$ das crianças encontravam-se na faixa de sobrepeso e obesidade (acima de 2,00 escores z).

Tabela 3: Distribuição dos escolares do gênero masculino, de acordo com o escore z de peso para a idade.

\begin{tabular}{|c|c|c|c|c|c|c|c|c|}
\hline \multicolumn{9}{|c|}{ ESCORE Z - PESO/IDADE } \\
\hline \multirow[t]{2}{*}{ IDADE } & \multicolumn{2}{|c|}{$<-2 Z$} & \multicolumn{2}{|c|}{-2 a $2 Z$} & \multicolumn{2}{|c|}{$>2 Z$} & \multicolumn{2}{|c|}{ TOTAL } \\
\hline & $\mathbf{N}$ & $\%$ & $\mathbf{N}$ & $\%$ & $\mathbf{N}$ & $\%$ & $\mathbf{N}$ & $\%$ \\
\hline 6 ANOS & - & 0 & 4 & 4,3 & 4 & 4,3 & 8 & 8,6 \\
\hline 7 ANOS & - & 0 & 14 & 15,05 & 4 & 4,3 & 18 & 19,35 \\
\hline 8 ANOS & 1 & 1,07 & 14 & 15,05 & 4 & 4,3 & 19 & 20,43 \\
\hline 9 ANOS & 2 & 2,15 & 23 & 24,73 & 2 & 2,15 & 27 & 29,03 \\
\hline 10 ANOS & 1 & 1,07 & 18 & 19,35 & 2 & 2,15 & 21 & 22,58 \\
\hline TOTAL & 4 & 4,29 & 73 & 78,48 & 16 & 17,2 & 93 & 100 \\
\hline
\end{tabular}


A Tabela 4 demonstra a distribuição das crianças do gênero feminino por meio do índice peso para a idade em escores z. Encontraram com déficit neste parâmetro (abaixo de
$-2,00$ escores z) 4 meninas (4,5\%), já na faixa de sobrepeso e obesidade (acima de 2,00 esco $\neg$ res $z$ ) encontravam-se 22 $(16,52 \%)$ das crianças.

Tabela 4: Distribuição dos escolares do gênero feminino, de acordo com o escore $\mathrm{z}$ de peso para a idade.

\begin{tabular}{|c|c|c|c|c|c|c|c|c|}
\hline \multicolumn{9}{|c|}{ ESCORE Z - PESO/IDADE } \\
\hline \multirow[t]{2}{*}{ IDADE } & \multicolumn{2}{|c|}{$<-2 Z$} & \multicolumn{2}{|c|}{-2 a $2 Z$} & \multicolumn{2}{|c|}{$>2 Z$} & \multicolumn{2}{|c|}{ TOTAL } \\
\hline & $\mathbf{N}$ & $\%$ & $\mathbf{N}$ & $\%$ & $\mathbf{N}$ & $\%$ & $\mathbf{N}$ & $\%$ \\
\hline 6 ANOS & - & 0 & 9 & 6,76 & 2 & 1,5 & 11 & 8,27 \\
\hline 7 ANOS & 1 & 0,75 & 26 & 19,54 & 7 & 5,26 & 34 & 25,56 \\
\hline 8 ANOS & 1 & 0,75 & 23 & 17,3 & 7 & 5,26 & 31 & 23,3 \\
\hline 9 ANOS & 2 & 1,5 & 28 & 21,05 & 5 & 3,75 & 35 & 26,31 \\
\hline 10 ANOS & 2 & 1,5 & 19 & 14,28 & 1 & 0,75 & 22 & 16,54 \\
\hline TOTAL & 4 & 4,5 & 105 & 78,93 & 22 & 16,52 & 133 & 100 \\
\hline
\end{tabular}

Na Tabela 5, está descrito a classificação das crianças do sexo masculino por meio do índice peso para a estatura, com déficit de estatura para a idade (escores $\mathrm{Z}$ inferior a $-2,00)$, encontrou-se $6(6,25 \%)$ dos meninos, $14(15,5 \%)$ encontravam acima de 2 escores $Z$.

Tabela 5: Distribuição dos escolares do gênero masculino, de acordo com o escore Z de peso para a estatura.

\begin{tabular}{|c|c|c|c|c|c|c|c|c|}
\hline \multicolumn{9}{|c|}{ ESCORE Z - PESO/ESTATURA } \\
\hline \multirow[t]{2}{*}{ IDADE } & \multicolumn{2}{|c|}{$<-2 Z$} & \multicolumn{2}{|c|}{-2 a $2 Z$} & \multicolumn{2}{|c|}{$>2 Z$} & \multicolumn{2}{|c|}{ TOTAL } \\
\hline & $\mathbf{N}$ & $\%$ & $\mathbf{N}$ & $\%$ & $\mathbf{N}$ & $\%$ & $\mathbf{N}$ & $\%$ \\
\hline 6 ANOS & - & 0 & 5 & 5,37 & 3 & 3,22 & 8 & 8,6 \\
\hline 7 ANOS & - & 0 & 15 & 16,12 & 3 & 3,22 & 18 & 19,35 \\
\hline 8 ANOS & 1 & 1,07 & 14 & 15,05 & 4 & 4,3 & 19 & 20,43 \\
\hline 9 ANOS & 4 & 4,3 & 21 & 22,58 & 2 & 2,15 & 27 & 29,03 \\
\hline 10 ANOS & 1 & 1,07 & 18 & 6,45 & 2 & 2,15 & 21 & 22,58 \\
\hline TOTAL & 6 & 6,45 & 73 & 78,49 & 14 & 15,05 & 93 & 100 \\
\hline
\end{tabular}

A Tabela 6, representa as crianças do sexo feminino por meio do índice peso para a estatura, como poderá ser observado na tabela abaixo $15(11,25 \%)$ de meninas estavam apresentando déficit de estatura para a idade, (escores $\mathrm{Z}$ inferior a -2,00), $22(16,52 \%)$ encontravam com escores $Z$ superior a 2 .

Tabela 6: Distribuição dos escolares do gênero feminino, de acordo com o escore Z de peso para a estatura.

\begin{tabular}{|c|c|c|c|c|c|c|c|c|}
\hline \multicolumn{9}{|c|}{ ESCORE Z - PESO/ESTATURA } \\
\hline \multirow[t]{2}{*}{ IDADE } & \multicolumn{2}{|c|}{$<-2 Z$} & \multicolumn{2}{|c|}{-2 a $2 Z$} & \multicolumn{2}{|c|}{$>2 Z$} & \multicolumn{2}{|c|}{ TOTAL } \\
\hline & $\mathbf{N}$ & $\%$ & $\mathbf{N}$ & $\%$ & $\mathbf{N}$ & $\%$ & $\mathbf{N}$ & $\%$ \\
\hline 6 ANOS & 1 & 0,75 & 10 & 7,51 & - & 0 & 11 & 8,27 \\
\hline 7 ANOS & 3 & 2,25 & 26 & 19,54 & 5 & 3,75 & 34 & 22,56 \\
\hline 8 ANOS & 3 & 2,25 & 22 & 16,54 & 6 & 4,51 & 31 & 23,3 \\
\hline 9 ANOS & 5 & 3,75 & 24 & 18,04 & 6 & 4,51 & 35 & 26,31 \\
\hline 10 ANOS & 3 & 2,25 & 14 & 10,52 & 5 & 3,75 & 22 & 16,54 \\
\hline TOTAL & 15 & 11,25 & 96 & 72,15 & 22 & 16,52 & 133 & 100 \\
\hline
\end{tabular}

A Tabela 7 representa o índice estatura para idade do gênero masculino. Foi possível observar que 3 (3,22\%) dos meninos estavam com baixa estatura para a idade (escore $\mathrm{z}$ inferior a-2,00). Com a estatura para idade acima da media (escore z superior a 2,00$)$ apenas $4(4,3 \%)$ dos meninos . 
Tabela 7: Distribuição dos escolares do gênero masculino, de acordo com o escore z de estatura para a idade.

\begin{tabular}{|c|c|c|c|c|c|c|c|c|}
\hline \multicolumn{9}{|c|}{ ESCORE Z - ESTATURA/IDADE } \\
\hline \multirow[t]{2}{*}{ IDADE } & \multicolumn{2}{|c|}{$<-2 Z$} & \multicolumn{2}{|c|}{-2 a $2 Z$} & \multicolumn{2}{|c|}{$>2 \mathrm{Z}$} & \multicolumn{2}{|c|}{ TOTAL } \\
\hline & $\mathbf{N}$ & $\%$ & $\mathbf{N}$ & $\%$ & $\mathbf{N}$ & $\%$ & $\mathbf{N}$ & $\%$ \\
\hline 6 ANOS & 1 & 1,07 & 5 & 5,37 & 2 & 2,15 & 8 & 8,6 \\
\hline 7 ANOS & - & 0 & 16 & 17,2 & 2 & 2,15 & 18 & 19,35 \\
\hline 8 ANOS & 1 & 1,07 & 16 & 17,2 & 2 & 2,15 & 19 & 20,43 \\
\hline 9 ANOS & 2 & 2,15 & 23 & 24,73 & 2 & 2,15 & 27 & 29,03 \\
\hline 10 ANOS & 3 & 3,22 & 14 & 15,05 & 4 & 4,3 & 21 & 22,58 \\
\hline TOTAL & 7 & 7,52 & 74 & 79,55 & 12 & 12,9 & 93 & 100 \\
\hline
\end{tabular}

Na Tabela 8, está descrito o índice estatura para idade do sexo feminino. A analise dessa tabela afirma que 12 (9\%) das meninas estavam com baixa estatura para a idade (escore z inferior a -2,00). $11(8,25 \%)$ das meninas estavam com a estatura para a idade superior ao desejado (escore $\mathrm{z}$ superior a 2,00).

Tabela 8: Distribuição dos escolares do gênero feminino, de acordo com o escore z de estatura para a idade.

\begin{tabular}{|c|c|c|c|c|c|c|c|c|}
\hline \multicolumn{9}{|c|}{ ESCORE Z - ESTATURA/IDADE } \\
\hline IDADE & \multicolumn{2}{|c|}{$<-2 Z$} & \multicolumn{2}{|c|}{-2 a $2 Z$} & \multicolumn{2}{|c|}{$>2 Z$} & \multicolumn{2}{|c|}{ TOTAL } \\
\hline & $\mathbf{N}$ & $\%$ & $\mathbf{N}$ & $\%$ & $\mathbf{N}$ & $\%$ & $\mathbf{N}$ & $\%$ \\
\hline 6 ANOS & 1 & 0,75 & 8 & 6,01 & 2 & 1,5 & 11 & 8,27 \\
\hline 7 ANOS & - & 0 & 32 & 24,06 & 2 & 1,5 & 34 & 5,56 \\
\hline 8 ANOS & 3 & 2,25 & 26 & 19,54 & 2 & 1,5 & 31 & 23,3 \\
\hline 9 ANOS & 4 & 3 & 29 & 21,8 & 2 & 1,5 & 35 & 6,31 \\
\hline 10 ANOS & 4 & 3 & 15 & 11,27 & 3 & 2,25 & 22 & 6,54 \\
\hline TOTAL & 12 & 9 & 100 & 82,68 & 11 & 8,25 & 133 & 100 \\
\hline
\end{tabular}

Foi possível identificar mediante de um questionário que $64(28,31 \%)$ crianças participantes da pesquisa praticam atividade física com frequência, relatam que é rotineira (3 a mais vezes por semana), $154(68,14 \%)$ relataram que praticam atividade física somente na escola durante a matéria curricular de Educação Física que acontece 2 vezes por semana, apenas $8(3,53 \%)$ disse não praticar nem um tipo de atividade física.

Constatou-se que 73 (32,3\%) crianças comem doces mais de três vezes por semana, 135 (59,73\%) relatou comer doces de uma a duas vezes por semana e 18 (7,96\%) disse não comer doces, sendo que 131 (57,96\%) têm preferências em comer doces industrializados e $77(34,07 \%)$ doces caseiros.
Foi perguntado para as crianças com que frequência elas comem lanches fora de casa, tendo como resposta que $36(15,92 \%)$ comem lanches fora de casa sempre (frequentemente), $169(74,77 \%)$ respondeu às vezes, e somente 21 $(9,29 \%)$ responderam não comer lanches fora de casa.

Na Figura 1A é mostrado como as crianças costumam permanecer ou brincar quando estão em suas residências, como pode ser notado as crianças desse estudo ainda brincam de correr, pedalar ou até mesmo nadar, representam $39,73 \%$ dos estudados, tendo como preferência as brincadeiras que permanece mais tempo sentado do que correndo $18,14 \%$ e soma um total de crianças que só assistem televisão ou jogam vídeo game um total de $42,12 \%$. 
Figura 1: Gráficos representativos quanto a respostas obtidas pelo questionamento aplicado aos alunos de 6 à 10 anos em Escola Estadual de Umuarama/PR. Figura A. Brincadeiras e forma de permanência das crianças quando estão em suas residências. Figura B. Número de refeições diárias. Figura C: Frequência do consumo de massas por semana. Figura D: frequência do consumo de verduras e legumes. Figura E: Como as crianças sentem quanto à discriminação em relação ao peso:

A

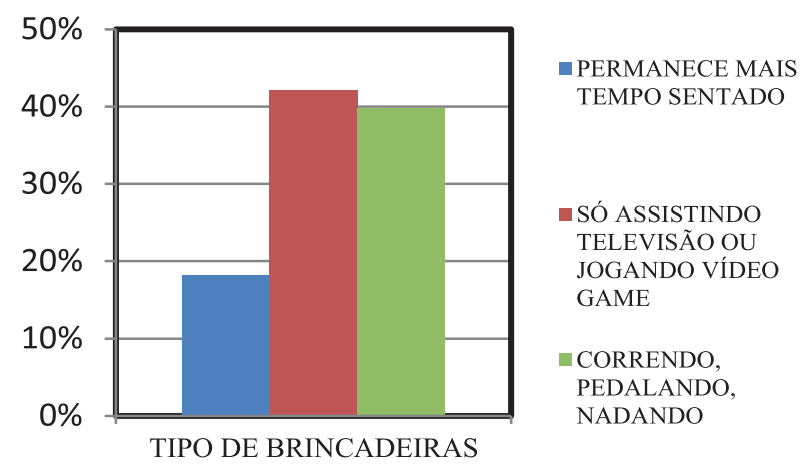

$\mathrm{C}$

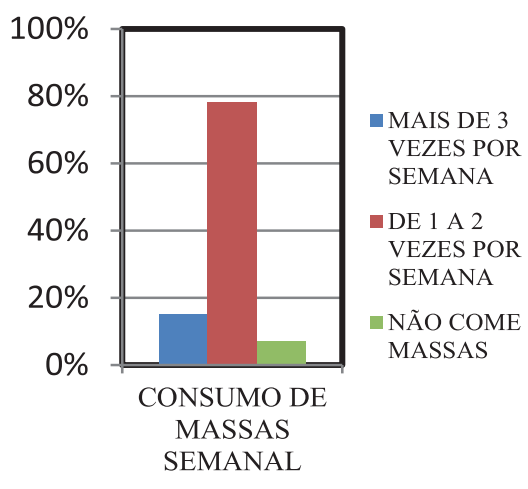

B

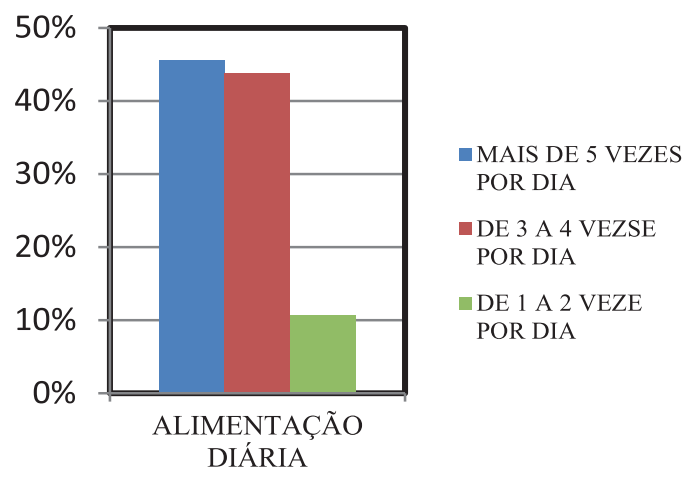

$\mathrm{E}$

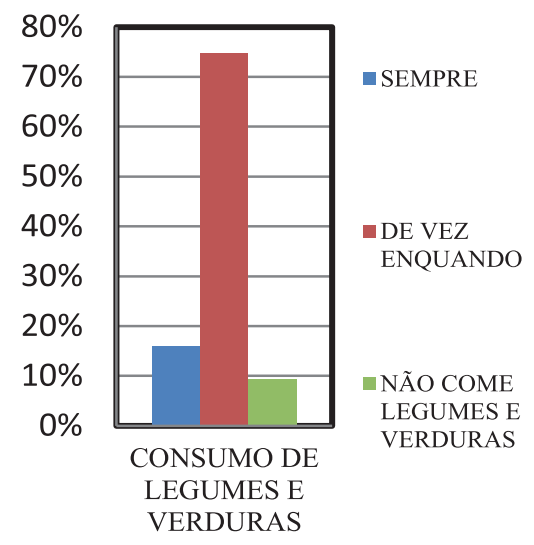

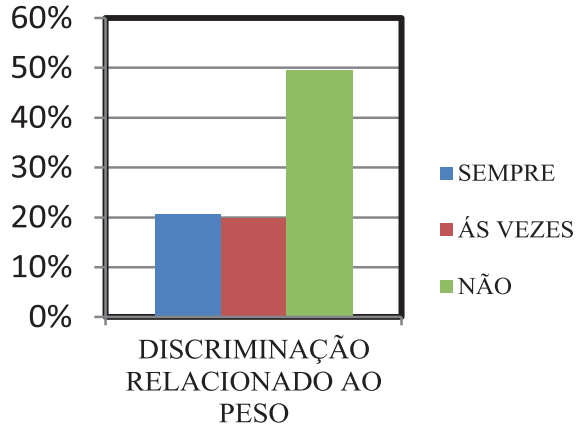

A Figura 1B apresenta os dados relacionados à alimentação diária das crianças participantes da pesquisa. Os dados observados revelam que $45,57 \%$ das crianças comem mais de cinco vezes por dia, 43,8 se alimentam de três a quatro vezes ao dia e $10,61 \%$ comem somente 1 ou duas vezes por dia. Os dados da Figura 1C revelam que o consumo de massas durante a semana é comum no cardápio das famílias, pois foi visto que $78 \%$ das crianças comem massas de uma a duas vezes por semana, 15,04 comem mais de três vezes por semana e 7,1 não consomem massas.

Como poderá ser observado na Figura 1D, o consumo de verduras e legumes pelas crianças não é rotina, pois foram encontrados $44,67 \%$ das crianças não tinham o habito constante de seu consumo, 15,48 não comiam de forma alguma esses alimentos, mas em contra partida 40,82 afirmam comer diariamente.Na figura 1E, está mostrando a porcentagem de crianças que sentem discriminação em relação ao peso, identificado através do questionário. Foi possível identificar que 49,46\% não sentem qualquer discriminação em relação ao peso, $19,91 \%$ às vezes sentem discriminação e $20,61 \%$ dizem sofrer discriminação sempre.

\section{Discussão}

No Brasil, a rápida diminuição das taxas de desnutrição associada ao aumento nas taxas de obesidade tem ocorrido em curto intervalo de tempo, agregando uma nova preocupação que diz respeito ao âmbito das políticas públicas, que envolve os cuidados alimentares e nutricionais com as crianças (FERNANDES; GALLO; ADVÍNCULA, 2006; DUCAN et al., 2011).

De acordo com a Organização Mundial de Saúde um dos mais importantes motivos para a classificação de sobrepeso e obesidade é permitir as comparações significantes do status de peso dentro e entre as populações, identificando indivíduos e grupos com maior risco de morbidade e mortalidade, estabelecendo prioridades para intervenção em níveis coletivo e individual e também dão embasamento para avaliar intervenções (OMS, 2004).

Para o desenvolvimento deste trabalho foi utilizado a antropometria para a avaliação do estado nutricional das crianças em questão, pois é considerado o método mais útil para rastrear obesidade, por ser barato, não invasivo, universalmente aplicável, e com boa aceitação pela população (VICTORA et al.,1998; ABRANTES et al., 2002).

Índices antropométricos são obtidos a partir da combinação de duas ou mais informações antropométricas básicas (peso, gênero, idade, altura). Esses índices em crianças, os mais utilizados são o peso/idade, a altura/idade e o peso/altura sendo que eles são obtidos comparando-se as informações de peso, altura, idade e gênero com curvas de referência, como a do National Center for Health Stastistics 
(NCHS) (NCHS, 1978; VICTORA et al., 1998; ABRANTES et al., 2002).

$\mathrm{Na}$ questão relacionada ao IMC, foi possível verificar que entre as crianças do gênero masculino 18,25\% apresentaram sobrepeso e $21,5 \%$ índice compatível com obesidade, já as meninas o resultado encontrado foi $16,54 \%$ e $23,3 \%$, respectivamente. Resultado semelhante ao encontrado em outros estudos, como o estudo realizado por Costa; Cintra; Fisberg (2006) que analisaram o IMC de crianças na faixa etária de 7 a 10 anos. No estudo supracitado observou-se uma prevalência de sobrepeso de $14,8 \%$ e obesidade de $20,3 \%$ para o sexo masculino e para o gênero feminino foi encontrado $16,6 \%$ de sobrepeso e obesidade contatou-se 15,8\%.

Quando comparado ao estudo realizado por Soar et al. (2004), em Florianópolis envolvendo crianças de 7 a 9 anos de idade, a presente pesquisa apresentou um índice mais elevado de obesidade em ambos os gêneros, pois os resultados encontrados por eles foi de $17,9 \%$ de sobrepeso e 6,7 de obesidade sendo sua prevalência no gênero masculino. $\mathrm{O}$ autor acredita que a maior prevalência de obesidade no gênero masculino, esteja ligada ao fato da aceleração do crescimento acontecer de forma mais tardia nesse sexo.

De acordo com Traebert et al. (2004); Castro (2005) o risco de uma criança obesa permanecer nesta condição na vida adulta é de $25 \%$, aumentando para $80 \%$ quando o excesso de peso se instala durante a adolescência. O sobrepeso e a obesidade contribuem de forma importante para a carga de doenças crônicas e incapacidades na vida adulta (ERDMANN, 2006; WANG et al., 2014).

Desta forma, pode-se notar na Figura 1A que $42,12 \%$ das crianças só assistem televisão ou jogam vídeo game, isso pode estar diretamente ligado ao resultado do IMC das crianças que foram classificadas como obesas, pois foi encontrado um total de $44,8 \%$, tendo como preferência as brincadeiras que permanecem mais tempo sentados do que correndo $18,14 \%$, porém $39,73 \%$ ainda brincam de correr, pedalar ou até mesmo nadar. O que se pode abstrair do comportamento das crianças nos dias atuais é que as mudanças no estilo de vida (permanência em frente à televisão, computador, vídeo game e dificuldades de brincar na rua pela falta de segurança) favorecem um balanço energético positivo, aumentando a prevalência da obesidade infantil (OLIVEIRA et al., 2003; PÁDUA et al., 2008; von HIPPEL; NAHHAS, 2013).

Em estudos realizados foi detectada associação significante entre horas despendidas com hábito de assistir TV e aumento das prevalências de sobrepeso e obesidade, e tal associação ocorre possivelmente em função da natureza sedentária da atividade, acrescida da relação que existe entre a mesma e o consumo de lanches e, também, ao efeito cumulativo da exposição a propagandas de alimentos hipercalóricos (OLIVEIRA et al., 2003; WILLIAMS et al., 2015).

Corroboram com esse entendimento Hancox; Poulton (2006), pois verificaram o impacto do hábito de assistir televisão no IMC e no sobrepeso em crianças e adolescentes com idade entre 3 e 15 anos e os resultados demonstraram que o tempo gasto assistindo televisão é um preditor importante do sobrepeso e aumento do IMC na infância. Portanto, fica evidenciado que os hábitos sedentários juntamente com o consumo de alimentos calóricos colaboram positivamente para o sobrepeso e a obesidade (HO et al., 2012).
Como já citado anteriormente o número de crianças que consome doce frequentemente (pelo menos $1 \mathrm{vez}$ na semana) é alto somando um total de $92,03 \%$, sendo que $57,96 \%$ preferem doces industrializados e também tem preferência por lanches de fácil acesso feitos fora de suas residências. Neste estudo foi verificado que $74,77 \%$ das crianças, às vezes, comem os lanches rápidos fora de casa e 15,92\% diz comer com frequência, 93,04\% das crianças estudas relataram comer massas pela menos 2 vezes por semana.No que se refere ao consumo de verduras e legumes não é muito constante para a grande maioria das crianças $(59,67 \%)$.Por isso, esses hábitos alimentares exemplificam bem a chamada dieta ocidental, que consiste em uma alimentação rica em gorduras, sobretudo as de origem animal, açúcar e alimentos refinados além de reduzida ingestão de carboidratos complexos e fibras, favorecendo o estoque energético (OLIVEIRA et al., 2003; MORELLO et al., 2012).

Viuniski (2007) complementa o disposto acima dizendo que o aumento da obesidade, esta relacionado com as grandes mudanças ambientais que ocorre juntamente com o progresso das últimas décadas, com as pessoas vivendo num estilo mais sedentário e tendo acesso a alimentos ricos em gordura, calorias e pobre em fibras.

De acordo com os dados obtidos através do questionário utilizado nesta pesquisa, verificou-se que $28,31 \%$ das crianças praticam atividade física com freqüência, (de 3 a mais vezes por semana) e $68,14 \%$ praticam atividade física somente na escola durante a matéria curricular de educação física, apenas 3,53\% não praticam atividade física alguma. Nesse sentido torna-se evidente a necessidade de ações preventivas desde as idades mais precoces (CRESPO et al., 2012). Dessa forma, a escola deve ocupar papel de destaque tanto em relação à orientação de hábitos alimentares quanto à prática de exercícios físicos (COSTA; CINTRA; FISBERG, 2006). Sendo assim Viuniski (2007), sugere que algumas atividades deveriam ser incluídas no dia a dia das crianças, como, exemplo, caminhadas, bicicleta, natação, dançar, pular corda, jogos de bola, brincadeiras ao ar livre entre outras com gasto energético.

Durante a pesquisa foi possível identificar que $33,72 \%$ das crianças apresentavam-se com peso superior ao recomendado para a idade e sexo e um total de $8,79 \%$ baixo peso, um número superior ao encontrado no estudo de Rezende et al., (2008) que analisaram o P/I de 346 crianças na faixa etária de 7 a 14 anos, onde identificou-se que 19,9\% apresentavam-se acima do peso ideal para idade e sexo sendo 12,21\% com sobrepeso, 7,69\% obesidade, e, com déficit de peso $5,81 \%$.

Quando analisado o escore $\mathrm{Z}$ peso/estatura a prevalência de déficit de peso para estatura foi de 17,7 , as crianças que apresentaram peso superior para sua estatura foi de $32,02 \%$. Observa-se um número superior de desnutrição e de casos de excesso de peso quando comparado ao estudo realizado em Belém por Neves et al. (2006), que envolveu 637 crianças de 6 a 9 anos, pois, os resultados encontrados de déficit de peso foi de $4,2 \%$ e com excesso foi $7,4 \%$.

Conforme observado nos escores $\mathrm{Z}$ E/I das crianças avaliadas neste trabalho, $12,9 \%$ dos meninos eram mais alta que o esperado para sua idade e com déficit nesse escores $Z$ foi encontrado $7,52 \%$, já as meninas $9 \%$ e $8,25 \%$ respectivamente. 
Fernandes; Gallo; Advíncula (2006) acreditam que o fator determinante para o aumento no crescimento das crianças esteja diretamente ligado com o melhor acesso das crianças e os familiares aos serviços de saúde, ao saneamento básico e melhoria nas condições de moradia, além do aumento educacional no país. Em contrapartida a deficiência do crescimento das crianças depende de vários fatores, tais como, ocorrência de doenças infectocontagiosa e alimentação inadequada (GUIMARÃES; BARROS, 2001). O estado nutricional de uma criança é fundamental para que seu crescimento seja progressivo e para que ela desenvolva suas aptidões psicomotoras e sociais (HURLEY; CROSS; HUGHES, 2011).

As alterações nutricionais tanto o déficit como o excesso trazem riscos potenciais de agravos à saúde, bem como a futuros problemas de relações interpessoais e funcionais dentro da comunidade (SANTOS; LEÃO, 2008), conforme dados já coletados, $20,61 \%$ das crianças sentem ser discriminadas constantemente devido ao seu peso, e 19,91\% às vezes sentem-se discriminada e esse ato discriminatório pode acarretar sérios problemas sociais e comportamentais envolvendo sua imagem; como transtornos psicológicos, depressão, ansiedade, auto-estima baixa e dificuldade de ajustamento fazendo com que as crianças discriminadas se isolem (LUIZ et al., 2005; ESCRIVÃO et al., 2000).

Ainda em relação a esse assunto Faria (2007), coloca que as crianças e adolescentes obesos são vítimas das mais diversas discriminações sociais, sujeitos a todo tipo de pilhérias, segregações e imagens irônicas, infelizmente oriundas principalmente de familiares e colegas de escola.

\section{Conclusão}

Tendo em vista o estudo realizado verificou-se que uma parcela razoável das crianças apresentara escores $\mathrm{Z}$ peso/estatura com alterações indicando sobrepeso e obesidade, geralmente ocasionado pelos hábitos alimentares errôneos e a falta de atividade física constante.

Sendo assim torna-se importante um maior incentivo para que haja uma melhora na qualidade e quantidade de atividade física e também orientações relacionadas à alimentação saudável, dessa forma evitando que essas crianças se tornem adultos obesos, podendo então diminuir os riscos de desenvolverem a Síndrome Metabólica nessa fase da vida, também com finalidade de evitar os problemas psicológicos gerados pela discriminação relacionada com o excesso de peso, que podem gerar ansiedade, depressão e até mesmo o isolamento social.Fica claro que o envolvimento familiar juntamente com a escola torna-se essencial para a prevenção e um tratamento da obesidade com êxito.

\section{Referências}

ABRANTES, M. M.; LAMOUNIER, J. A.; COLOSIMO, E. A. Prevalência de sobrepeso e obesidade em crianças e adolescentes das regiões Sudeste e Nordeste. J. Pediatr., v. 78 , n. 4 , p. $335-340,2002$.

BARBOSA, V. L. P. Prevenção da obesidade na infância e na adolescência: exercício, nutrição e psicologia. São Paulo: Manole, 2008, 184p.
BOUGLÉ, D.; BROUGARD, J. Iron in child obesity: Relationships with inflammation and metabolic risk factors. Nutrients, v.19, n.6, p. 2222-2230, 2013.

CASTRO, T. G. et al. Caracterização do consumo alimentar, ambiente sócio econômico e estado nutricional de préescolares de creches municipais. Rev. Nutr., v. 18, n. 3, p. 321-330, 2005.

CHEN, W.; BERENSON, G. S. Síndrome metabólica: definição e prevalência em crianças. J. Pediatr, v. 83, n. 1, p. 1-3, 2007.

CIOLAC, E. G.; GUIMARÃES, G. V. Exercício físico e síndrome metabólica. Rev. Saúde Pública, v. 10, n. 4, p. 325-330, 2004.

COSTA, R. F. da; CINTRA, I. de P.; FISBERG, M. Prevalência de sobrepeso e obesidade em escolares da cidade de Santos, SP. Arq. Bras. Endocrinol. Metab., v. 50 , n. 1, p. 60-67, 2006.

CRESPO, N. C. et al. Results of a multi-level intervention to prevent and control childhood obesity among Latino children: the Aventuras Para Niños Study. Ann. Behav. Med., v. 43, n. 1, p. 84-100, 2012.

DIETZ, W. H. Childhood obesity. In: SHILS, M. E. Modern Nutrition in Health and Disease. Baltimore: Williams \& Williams., 1999, p. 1071-1080.

DUCAN, S. et al. Modifiable risk factors for overweight and obesity in children and adolescents from São Paulo, Brazil. BMC Public Health, v. 11, n. 585, p. 1-9, 2011.

ERDMANN, A. Efeito da suplementação de zinco em indivíduos obesos submetidos à cirurgia bariátrica. 2006. 124 f. Dissertação (Mestrado em Nutrição) Departamento de Nutrição - Universidade Federal de Santa Catarina, Florianópolis, 2006.

ESCRIVÃO, M. A. M. S. et al. Obesidade exógena na infância e na adolescência. Jornal de Pediatria, v. 76, n. 3, p. 305-S310, 2000.

Expert Panel on Detection, Evaluation, and Treatment of High Blood Cholesterol in Adults. Adult Treatment Panel III. Executive Summary of the Third Report of The National Cholesterol Education Program (NCEP). JAMA. p. 285: 2486-2497, 2001.

FARIA, E. V. Características nutricionais e fatores associados a sobrepeso e obesidade em adolescentes tendidos em ambulatório da faculdade de ciências médicas de minas gerais em belo horizonte. 2007. 162 f. Dissertação (Mestrado em Medicina) - Faculdade de Medicina. Universidade Federal de Minas Gerais,, Belo Horizonte, 2007.

FISBERG, M. et al. Obesidade na infância e adolescência. São Paulo: Fundação Byk; 1995, 218p. 
FERNANDES, I. T.; GALLO, P. R.; ADVINCULA, A. O. Avaliação antropométrica de pré-escolares do município de Mogi-Guaçú, São Paulo: subsídio para políticas públicas de saúde. Rev. Bras. Saúde Mater. Infant, v. 6, n. 2, p. 551558,2006

FERREIRA, A. P.; OLIVEIRA, C. E. R.; FRANCA, N. M. Síndrome metabólica em crianças obesas e fatores de risco para doenças cardiovasculares de acordo com a resistência à insulina (HOMA-IR). J. Pediatr., v. 83, n. 1, p. 21-26, 2007.

FRIEDRICH, R. R. et al. Design, randomization and methodology of the TriAtiva Program to reduce obesity in school children in Southern Brazil. BMC Public Health, v. 11, n. 1, p. 15-363, 2015.

GUIMARAES, L. V.; BARROS, M. B. A. As diferenças de estado nutricional em pré-escolares de rede pública e a transição nutricional. J. Pediatr., v. 77, n. 5, p. 381-386, 2001.

HALPERN, Z. S. C.; RODRIGUES, M. D. B,; COSTA, R. F. Determinantes fisiológicos docontrole do peso e apetite. Rev. Psiq. Clin., v. 31, n. 4, p. 150-153, 2004.

HANCOX, R. J.; POULTON, R. Watching television is associated with childhood obesity: but is it clinically important? Int. J. Obes., v. 30, n. 1, p. 171-75, 2006.

HEPTULLA, R. et al. Temporal patterns of circulating leptin levels in lean and obese adolescents: relationships to insulin, growth hormone, and free fatty acids rhythmicity. $\mathbf{J}$ Clin. Endocrinol. Metab., v. 86, n. 1, p. 90-96, 2001.

HO, M. et al. Effectiveness of lifestyle interventions in child obesity: systematic review with meta-analysis. Pediatrics, v. 130, n. 6 , p. 1647-1671, 2012.

HUGHES, S. O. et al. Executive functioning, emotion regulation, eating self-regulation, and weight status in lowincome preschool children: how do they relate? Appetite, v. 89, n. 1, p. 1-9, 2015.

HURLEY, K. M.; CROSS, M. B.; HUGHES, S. O. A systematic review of responsive feeding and child obesity in high-income countries. J Nutr, v. 141, n. 3, p. 495-501, 2011

LEE, A. et al. Prediction of future risk of insulin resistance and metabolic syndrome based on Korean boy's metabolite profiling. Obes. Res. Clin. Pract., v. 9, n. 4, p. 336-345, 2014

LEMOS, M. L. C. et al. Obesidade na infância e adolescência: critérios de diagnóstico clínico e laboratorial. Rev. Pediatr., v. 8, n. 1, p. 8-16, 2007.

LERARIO, D. D. G. et al. Excesso de peso e gordura abdominal para a síndrome metabólica em nipo-brasileiros. Rev. Saúde Pública, v. 36, n. 1, p. 4-11, 2002.
LOTTENBERG, S. A.; GLEZER, A.; TURATTI, L. A. Síndrome metabólica: identificando fatores de risco. J. Pediatr., v. 83, n. 5, p. S204-S208, 2007.

LUCA, A. C.; IORDACHE, C. Obesity - A risk factor for cardiovascular diseases. Rev. Med. Chir. Soc. Med. Nat. Iasi., v. 117, n. 1, p. 65-71, 2013.

LUIZ, A. M. A. G. et al. Depressão, ansiedade e competência social em crianças obesas. Estud. Psicol., v. 10, n. 1, p. 35-39, 2005.

MONDINI, L. et al. Prevalência de sobrepeso e fatores associados em crianças ingressantes no ensino fundamental em um município da região metropolitana de São Paulo, Brasil. Cad. Saúde Pública, v. 23, n. 8, p. 1825-1834, 2007.

MORELLO, M. I. et al. Associations among parent acculturation, child BMI, and child fruit and vegetable consumption in a Hispanic sample. J Immigr. Minor. Health, v. 14, n. 6, p. 1023-1029, 2012.

MÜLLER, R. C. L. Medicina do adolescente. São Paulo: Sarvier, p. 90-104, 2003.

NATIONAL CENTERS FOR HEALTH STATISTICS (NCHS). "Growth curves for children, birth - 18 years". Hyattsville, NCHS, (DHEW (PHS) 78.1650-Series 11, 165, 1978.

NEVES, O. M. D. das. et al. Antropometria de escolares ao ingresso no ensino fundamental na cidade de Belém, Pará, 2001. Rev. Bras. Saude Mater. Infant., v. 6, n. 1, p. 39-46, 2006.

ORGANIZAÇÃO MUNDIAL DA SAÚDE. Prevenindo e Controlando a Epidemia Global. São Paulo. Roca, 2004

OLIVEIRA, C. L. de; FISBERG, M. Obesidade na infância e adolescência: uma verdadeira epidemia. Arq. Bras. Endocrinol. Metab., v. 47, n. 2, p. 107-108, 2003

OLIVEIRA, A. M. A. de. et al. Sobrepeso e obesidade infantil: influência de fatores biológicos e ambientais em Feira de Santana, BA. Arq. Bras. Endocrinol. Metab., v. 47, n. 2, p. 144-150, 2003

OLIVEIRA, A. M. A.; CERQUEIRA, E. M. M.; OLIVEIRA, A. C. Prevalência de sobrepeso e obesidade infantil na cidade de Feira de Santana-BA: detecção na família x diagnóstico clínico. J. Pediatr., v. 79, n. 4, p. 325328, 2003.

OLIVEIRA, C. L. et al. Obesidade e síndrome metabólica na infância e adolescência. Rev. Nutr., v. 17, n. 2, p. 237 $245,2004$.

PÁDUA, E. C. R. et al. Diagnóstico do estado nutricional de crianças de 6 - 10 anos em escolas da rede privada de ensino da cidade de São Paulo. 2008. $21 \mathrm{f}$. 
Monografia (Instituto de Pesquisa e Ensino Albert Einstein) Universidade Federal de São Paulo, São Paulo, 2008.

PÉREZ, R. C. Current mapping of obesity. Nutr. Hosp., v. 28, n. 5, p. 21-31, 2013.

REZENDE, V. A. et al. Prevalência de sobrepeso e obesidade em alunos de uma escola de rede pública de Anápolis. In: iniciação cientifica da Faculdade de Anhanguera de Anápolis, 2008, Anápolis. Anuário de produção de iniciação científica discente. $v$. XI, $n^{\circ} 12, p$ 203-213, 2008.

RIBEIRO, F. et al. Gordura visceral e síndrome metabólica: mais que uma simples associação.Arq. Bras. Endocrinol. Metab., v. 50, n. 2, p. 230-238, 2006.

SABIA, R. V.; SANTOS, J. E.; RIBEIRO, R. P. P. Efeito da atividade física associada à orientação alimentar em adolescentes obesos: comparação entre o exercício aeróbio e anaeróbio. Rev. Bras. Med. Esporte, v. 10, n. 5, p. 349355, 2004.

SANTOS, A. L. B.; LEAO, L. S. C. S. Anthropometric profile of preschool children of a day-care center in Duque de Caxias, Rio de Janeiro, Brazil. Rev. Paul. Pediatr., v. 26, n. 3, p. 218-224, 2008.

SOAR, C. et al. Prevalência de sobrepeso e obesidade em escolares de uma escola pública de Florianópolis, Santa Catarina. Rev. Bras. Saude Mater. Infant., v. 4, n. 4, p. 391-397, 2004.

SOUZA LEÃO, L. S. C. et al. Prevalence of obesity in school children from Salvador, Bahia. Arq Bras Endocrinol Metab, v. 47, n. 2, p. 151-157, 2003.

TATE, E. B. et al. Two facets of stress and indirect effects on child diet through emotion-driven eating. Eat. Behav., v. 18, n. 1, p. 84-90, 2015.

TRAEBERT, J. et al. Transição alimentar: problema comum à obesidade e à cárie dentária. Rev. Nutr, v. 17, n. 2, p. 247 253, 2004.

VICTORA, C. G. et al. Estimativa da prevalência de déficit de altura/idade a partir da prevalência de déficit de peso/ idade em crianças brasileiras. Rev. Saúde Pública, v. 32, n. 4, p. 321- 327, 1998.

VIUNISKI, N. Pontos de Corte de IMC Para Sobrepeso e Obesidade em Crianças e Adolescentes. ABESO. 3. Ed. Disponível em: <http://www.abeso.org.br>. Acesso em: 10 jun. 2016.

VON HIPPEL, P. T.; NAHHAS, R. W. Extending the history of child obesity in the United States: The fels longitudinal study, birth years 1930-1993. Obesity (Silver Spring), v. 10, n. 1, p. 2153-2156, 2013.

WANG, X. Y. et al. The impacts of obesity and metabolic abnormality on carotid intima-media thickness and nonalcoholic fatty liver disease in children from an Inland Chinese City. J. Clin. Med., v. 3, n. 1, p. 323-33, 2014.

WELSBY, D. et al. Process evaluation of an up-scaled community based child obesity treatment program: NSW Go4Fun ${ }^{\circledR}$. BMC Public Health, v. 14, n. 140, p. 2-8, 2014.

WHO. Report of a "WHO Consultation on Obesity. Defining the problem of overweight and obesity". In: Obesity, preventing and managing the global epidemic. Geneva, 1998.

WILLIAMS, J. et al. Associations between Food Outlets around Schools and BMI among Primary Students in England: A Cross-Classified Multi-Level Analysis. PLoS One, v. 10, n. 7, p. 1-17, 2015.

Recebido em: 07/04/2016 Aceito em: 10/05/2016 